\title{
REFLUXO LARINGOFARÍNGEO E BULIMIA NERVOSA: ALTERAÇÕES VOCAIS E LARÍNEGAS
}

\author{
Laryngopharyngeal reflux and bulimia nervosa: \\ laryngeal and voice disorders
}

\author{
Carla Aparecida Cielo ${ }^{(1)}$, Dayane Domeneghini Didoné(2), Enma Mariángel Ortiz Torres ${ }^{(3)}$, \\ Joziane Padilha de Moraes Lima ${ }^{(4)}$
}

\begin{abstract}
RESUMO
Tema: características da bulimia nervosa (BN) e do Refluxo Laringofaríngeo (RLF) e alterações vocais e laríngeas que podem decorrer da exposição da laringe ao ácido gástrico. Objetivo: descrever, por meio de revisão de literatura, as características do RLF e da BN, relacionando-os com as alterações vocais e laríngeas que podem se originar da exposição da laringe ao ácido gástrico que ocorre em ambas as condições. Conclusão: a BN se caracteriza por um transtorno alimentar de causa principalmente psicológica em que ocorre ingestão compulsiva de grande quantidade de alimentos, seguida de episódios de vômitos auto-induzidos com a finalidade de evitar o ganho de peso. O RLF consiste em uma variação clínica da DRGE, na qual o fluxo retrógrado do alimento e do ácido gástrico entra em contato com a laringe e suas estruturas. Em comum, a BN e o RLF apresentam este mecanismo de regurgitação que pode atingir a laringe. Dentre os sinais e sintomas laríngeos e vocais decorrentes da regurgitação do ácido gástrico sobre a laringe estão: globus faríngeo, disfonia, rouquidão, tosse seca, disfagia, halitose, lesões inflamatórias nas pregas vocais, laringite, amigdalite, edema e eritema glótico posterior e ou de aritenóides, da região retrocricóidea e interaritenóidea, estenose subglótica, irregularidades na mucosa das pregas vocais, hipertrofia de mucosa laríngea, leucoplasia, carcinoma, laringoespasmos, granulomas, úlcera de contato, nódulos vocais, pólipos vocais, edema difuso, granuloma, fixação uni ou bilateral de aritenóides, edema de Reinke, laringomalácia, estridor, e odinofagia.
\end{abstract}

DESCRITORES: Bulimia Nervosa; Mucosa Laríngea; Refluxo Larigofaríngeo; Transtornos da Alimentação; Voz

(1) Fonoaudióloga; Professora Adjunta do Curso de Graduação em Fonoaudiologia e do Programa de Pós Graduação em Distúrbios da Comunicação Humana da Universidade Federal de Santa Maria, UFSM, Santa Maria, RS, Brasil; Doutora em Linguística Aplicada pela Pontifícia Universidade Católica do Rio Grande do Sul.

(2) Aluna do Curso de Graduação em Fonoaudiologia da Universidade Federal de Santa Maria, UFSM, Santa Maria, RS, Brasil.

(3) Aluna do Curso de Graduação em Fonoaudiologia da Universidade Federal de Santa Maria, UFSM, Santa Maria, RS, Brasil.

(4) Aluna do Curso de Graduação em Fonoaudiologia da Universidade Federal de Santa Maria, UFSM, Santa Maria, RS, Brasil.

Conflito de interesses: inexistente

\section{INTRODUÇÃO}

Bulimia Nervosa (BN) é um distúrbio de comportamento caracterizado pela ingestão compulsiva de grande quantidade de alimentos, com pouco ou nenhum prazer, seguido de vômitos auto-induzidos com a finalidade de evitar o ganho de peso ${ }^{1-7}$.

O DSM-IV (1994) descreve a compulsão com base em dois aspectos:

1) ingestão, em um período limitado de tempo (por ex., dentro de um período de duas horas) de uma quantidade de alimentos definitivamente maior do que a maioria das pessoas consumiria durante um período similar e sob circunstâncias similares e; 
2) um sentimento de falta de controle sobre o comportamento alimentar durante o episódio (por ex., um sentimento de incapacidade de parar de comer ou de controlar que ou o quanto está comendo) ${ }^{3}$.

Esse mecanismo de regurgitação assemelha-se ao Refluxo Laringofaríngeo (RLF), sendo este uma variação clínica da Doença do Refluxo Gastroesofágico (DRGE), em que o fluxo retrógrado do alimento e do suco gástrico, através do esôfago, pode atingir as estruturas laringofaríngeas ${ }^{8-10}$. Como a estrutura tecidual laríngea não está apta a receber o ácido gástrico, várias lesões nesta área podem ocorrer levando a alterações da voz em maior ou menor grau.

No entanto, há carência expressiva de estudos na área da Fonoaudiologia que tenham pesquisado as conseqüências diretas da BN sobre a laringe e a voz, havendo maior número de trabalhos que buscam esta relação com o RLF.

Deste modo, parece possível que alterações laríngeas e vocais decorrentes da BN possam apresentar aspectos em comum com as alterações laríngeas e vocais ocasionadas pelo RLF.

Com base no exposto, o objetivo deste trabalho é descrever, por meio de revisão de literatura, as características do RLF e da BN, relacionando-os com as alterações vocais e laríngeas que podem se originar da exposição da laringe ao ácido gástrico que ocorre em ambas as condições.

\section{MÉTODO}

Foi realizada revisão da literatura sobre o tema proposto utilizando-se livros, monografias, artigos de periódicos científicos e internet. Nesta última, foram pesquisados artigos em base de dados do Lilacs, Bireme, Medline e Scielo no período de 1998 a 2009. A consulta foi realizada em fontes da literatura científica nacional e internacional. Para a busca, foram utilizadas as palavras-chave: bulimia, refluxo gastroesofágico (RGE), refluxo laringofaríngeo, alterações vocais, alterações laríngeas, e transtornos alimentares.

Os critérios de inclusão adotados para a seleção dos materiais bibliográficos foram: apresentar abordagens a respeito do RLF, da DRGE e ou da BN, alterações vocais e alterações laríngeas por RLF, DRGE ou por BN; correlacionadas ou não; apresentar data de publicação dentro do período dos últimos 12 anos (1998 a 2009).

Os critérios de exclusão dos materiais bibliográficos para esta pesquisa compreenderam: abordagem sobre alterações vocais e alterações laríngeas decorrentes de outras causas que não o RLF, a DRGE ou a BN; data de publicação anterior ao ano de 1998.

A partir da pesquisa realizada com os unitermos e com os critérios adotados, foram selecionadas 55 referências bibliográficas, sendo que, dessas, são duas monografias, nove livros nacionais, 36 artigos nacionais, e oito artigos internacionais.

Salienta-se que foram encontrados na literatura apenas três artigos relacionando a BN com alterações laríngeas, não sendo encontrados artigos sobre a possível relação do RLF com a BN.

\section{REVISÃO DA LITERATURA}

\section{Bulimia nervosa}

Galeno, médico e filósofo grego nascido no ano 130, descreveu pela primeira vez o termo bulimia como kynos orexia, que significa fome canina, a qual considerou consequência de um estado de ânimo anormal. Nos anos 1970, esse transtorno alimentar era conhecido como Bulimarexia e, a partir dos anos 1980, o termo que passou a ser utilizado foi o de BN. Assim, etimologicamente, a palavra "bulimia" se origina do grego bous: boi e limos: fome ${ }^{7,11}$.

$\mathrm{Na}$ atualidade, a valorização excessiva dada ao corpo delgado da mulher parece estar ligada ao aumento da ocorrência de transtornos alimentares como a anorexia e a BN. A pressão cultural, bem como a mídia e o imaginário coletivo, estimula a busca de um corpo perfeito à base de sacrifício, principalmente no que se refere à população feminina ${ }^{7,11,12}$.

Os métodos mais utilizados pelos bulímicos para manter o corpo idealizado são vômitos autoinduzidos (em mais de $90 \%$ dos casos), em que podem provocar mais de 20 episódios diariamente, uso de diuréticos, laxantes e inibidores de apetite, dietas e exercícios físicos ${ }^{5,6,11}$. O consumo abusivo de cafeína e/ou uso de cocaína também tem sido relatados na literatura ${ }^{6}$. Portanto, a BN poderia ser conceituada como um método de prevenir absorção calórica por purgação, instalando-se o ciclo "dieta à episódio de compulsão alimentar à purgação", que ilustra o fluxo da patologia alimentar ${ }^{13}$.

O padrão alimentar, na doença, caracterizase pela insistência dos pacientes em realizar uma dieta, na qual ocorre a ingestão de quantidades extremamente reduzidas de alimentos, sem valor nutricional. Com isso, o episódio bulímico pode ser desencadeado e o valor nutricional ingerido é elevado ${ }^{11,13}$. No período de compulsão alimentar, a ingestão média é de três a quatro mil calorias por episódio, mas já foram descritos episódios com uma ingestão de até 20 mil calorias ${ }^{14}$, sendo que a ingestão média normal de calorias para os homens 
é de 1.800 e 1.300 para as mulheres, sendo esta média dependente da taxa de metabolismo basal, do efeito térmico do alimento e da atividade física ${ }^{15}$.

Segundo o DSM-IV (1994), a BN se divide em dois subtipos clínicos: o purgativo, no qual há ingestão de laxantes e diuréticos, além de mecanismos de vômitos auto-induzidos, e um segundo subtipo, em que os pacientes realizam somente jejuns e atividades físicas excessivas ${ }^{11}$.

A percepção e os mecanismos de interação envolvidos na regulação do comportamento de ingestão alimentar geralmente encontram-se alteradas nos pacientes bulímicos. Assim, a detecção da fome e o controle da saciedade também são deficientes. Tais pacientes caracterizam-se, ainda, por comportamentos alimentares perturbados como expressiva repugnância, ingestão anormalmente lenta, e um "comer social" prejudicado ${ }^{13}$.

A BN é o mais comum dos transtornos alimentares, com prevalência de 1 a $2 \%$ entre mulheres jovens, sendo que, em relação ao sexo, ocorre em uma proporção de 10:1 em favor do sexo feminino, e a idade média de ocorrência se dá em torno dos 25 anos $^{2,5,11}$. A incidência dessa desordem aumenta com a melhoria da condição socioeconômica ${ }^{2}$.

Pode-se considerar a BN como multifatorial, pois são muitos os fatores que podem ser considerados etiológicos, como componentes biológicos, psicológicos, familiares e sócio-culturais ${ }^{11,12,16,17}$. Do ponto de vista biológico, pode ocorrer alteração no hipotálamo no sentido de não produzir mecanismos cerebrais de saciedade. O que se discute na literatura é se essa disfunção é primária ou secundária à própria $\mathrm{BN}$ e, caso seja secundária, se é devida a déficit nutricional ou a alteração emocional ${ }^{12}$. Os fatores psicológicos podem estar associados à depressão ${ }^{16}$, mas são várias as hipóteses psicológicas que tentam explicar o fenômeno da inanição.

Existe consenso entre as teorias de que os distúrbios de alimentação funcionam como uma espécie de contenção psicológica ao interromper o desenvolvimento físico das adolescentes, remetendo-as ao estado pré-puberal ${ }^{12}$. A influência genética também tem sido investigada, encontrando-se maior incidência de BN entre os gêmeos monozigóticos e mostrando uma taxa de hereditariedade de 45 a $55 \%{ }^{12}$.

Para caracterizar esta patologia, os episódios de compulsão alimentar e os métodos compensatórios utilizados devem ocorrer pelo menos duas vezes por semana, num tempo mínimo de três meses ${ }^{5}$.

Quanto ao tratamento desta desordem alimentar, pode-se citar a reabilitação nutricional, que visa, inicialmente, à redução dos episódios de dieta, da compulsão alimentar e do uso de métodos purgativos; a intervenção psicológica; e o tratamento medicamentoso, em que os antidepressivos são inicialmente efetivos ${ }^{18}$, pois parecem exercer seus efeitos terapêuticos através da ação central, em vias noradrenérgicas e serotoninérgicas ${ }^{19}$, além de reduzirem a frequência de sintomas como ansiedade, depressão e obsessão ${ }^{18}$. Em estudo realizado, verificou-se que os índices de remissão dos episódios bulímicos através do uso de antidepressivos foram maiores $(19,2 \%)$ em relação aos do uso do placebo (8\%). Na avaliação de 16 pacientes tratados com $100 \mathrm{mg} / \mathrm{dia}$ de milnaciprano, dez apresentaram redução em pelo menos 50\% dos sintomas, sendo que três tiveram remissão completa ao fim do estudo (após oito semanas) ${ }^{20}$.

\section{Doença do refluxo gatroesofágico, refluxo gastroesofágico e refluxo laringofaríngeo}

A DRGE é a doença esofágica mais comum 21,22 e pode ser definida como uma afecção crônica decorrente do fluxo retrógrado de parte do conteúdo gastroduodenal para o esôfago e/ou órgãos adjacentes, os quais não possuem mucosa adequada para recebê-lo ${ }^{9}$, acarretando vários sinais e sintomas esofágicos ou extra-esofágicos e até mesmo gerando lesões teciduais ${ }^{11,23-26}$. Dentre os conteúdos refluídos, o ácido clorídrico e a pepsina são os principais ${ }^{11,27}$.

Basicamente, quatro mecanismos fisiológicos protegem a via aérea superior das alterações do fluxo retrógrado de parte do conteúdo gastroduodenal para o esôfago e/ou órgãos adjacentes, salientando-se o esfíncter esofágico superior, constituído principalmente pelo músculo cricofaríngeo e uma pequena porção de fibras musculares do esôfago distal ${ }^{9,21,28-30}$ e o inferior ${ }^{9,21,31,32}$ que, durante a deglutição, apresentam um mecanismo sincronizado de abertura e fechamento, com função de prevenir o escape do alimento do estômago ou do esôfago para as regiões superiores (laringe, faringe, cavidades oral e nasal) ${ }^{9}$. Além desses, a mucosa esofágica e as ondas motoras do esôfago também fazem parte do mecanismo de proteção ${ }^{28}$.

Para prevenir o refluxo, o esfíncter esofágico inferior, que está localizado no nível do hiato diafragmático, e que atua como principal barreira ${ }^{21}$ deve manter uma pressão maior do que a do estômago. Quando esse equilíbrio não é mantido, a pressão gástrica torna-se maior do que a do próprio esfíncter esofágico inferior, ocorrendo, então, a regurgitação, sendo que a diminuição da pressão do esfíncter esofágico inferior pode ocorrer por afrouxamento muscular, hérnia de hiato ou por relaxamento transitório ${ }^{9}$.

A DRGE é de grande importância médico-social por determinar sintomas de intensidade variável e que pode se manifestar de modo prolongado, 
podendo prejudicar a qualidade de vida do paciente. A prevalência é grande e estima-se que $40 \%$ da população adulta apresentarão refluxo em alguma fase da vida ${ }^{24,33}$.

Dados de estatísticas americanas revelam que a frequência anual dos sintomas típicos da DRGE é de $58,7 \%$ e a semanal de $19,8 \%$, sendo que no Canadá, a ocorrência semanal desses sintomas é de $30 \%$. Na Europa, somente a Finlândia tem frequência tão elevada (30\% semanal). Outros países como a Inglaterra e a Suécia, têm ocorrência mensal em torno de $18 \%$ e $25 \%$, respectivamente ${ }^{34}$.

A DRGE também pode apresentar sintomatologia exclusivamente extra-esofágica, laringofaríngea e/ou traqueo-brônquica, caracterizando, assim, o RLF ${ }^{25}$ que pode atingir a laringe e as pregas vocais, com consequências sobre a voz. Os pacientes do sexo masculino apresentam maior incidência de alterações laríngeas que os do sexo feminino ${ }^{9}$, apesar de a DRGE predominar no sexo feminino ${ }^{35}$.

$\mathrm{Na}$ realização do diagnóstico da DRGE, é importante uma anamnese bem detalhada sobre os principais sinais e sintomas e, além disso, para melhor caracterização da doença, aconselha-se a realização da endoscopia, Phmetria ${ }^{9,21}$ manometria, laringoscopia ${ }^{21}$ e a análise vocal perceptivoauditiva nos pacientes que referirem queixas e sintomas vocais ${ }^{9}$. Na laringoscopia, a DRE aparece em 40 a $75 \%$ de pacientes com sintomas laríngeos e evidências de patologias laríngeas na laringoscopia. Contudo, cerca de 20 a $40 \%$ dos pacientes com sintomas laríngeos não apresentam modificações no exame laringoscópico ${ }^{21}$.

A fisiopatologia do RGE difere do RLF, pois o primeiro apresenta falta de mobilidade e disfunção do esfíncter esofágico inferior, enquanto os indivíduos com sintomas de cabeça e pescoço têm boa mobilidade do esfíncter esofágico superior, mas disfunção do mesmo. Além disso, pacientes com RGE usualmente apresentam refluxo quando estão em posição supino ${ }^{21}$ e os com RLF apresentam refluxo durante o dia, em posição vertical ${ }^{21,36}$.

O primeiro estudo que distinguiu o RLF do RGE constatou que dos indivíduos que tinham RLF, $87 \%$ apresentavam pigarro, contrariamente aos que tinham RGE, que referiam este sintoma em apenas $3 \%$ dos casos. Por outro lado, em relação à pirose, ou sensação de queimação, $83 \%$ dos pacientes com RGE a referiam contra apenas $20 \%$ dos pacientes com RFL ${ }^{28}$. O RGE acomete o esôfago distal sem vômitos ${ }^{8,21}$, enquanto o RLF acomete o terço proximal do esôfago, a laringe ${ }^{25} \mathrm{e}$, em alguns casos, pode chegar à rinofaringe. Portanto, RGE e RLF são variações clínicas diferentes da DRGE ${ }^{8}$.
A primeira associação entre doença laríngea e RLF foi relatada por Coffin em $1903^{37}$, demonstrando que a "eructação de gases do estômago" e a hiperacidez eram responsáveis por sintomas em muitos de seus pacientes com "catarro pós-nasal". Estudos subsequentes afirmam que a mucosa da laringe é bastante sensível a substâncias químicas e que mesmo RLF intermitentes podem causar graves lesões inflamatórias 11,27,35,38, pois, ao contrário do esôfago, pouco se conhece a respeito dos fatores mecânicos e químicos que atuam na sua proteção ${ }^{35}$.

Como o tratamento do RGE deve ser efetivo, torna-se necessário que o paciente não o interrompa, pois deve ser realizado de modo continuado e prolongado com os objetivos de eliminação dos sintomas, cicatrização da mucosa, tratamento de complicações e manutenção do refluxo assintomático ${ }^{9}$

São essenciais no tratamento algumas estratégias como: elevação da cama realizada sob os pés da cabeceira ou embaixo do colchão, procurando evitar a elevação da cabeça com travesseiros, pois isto pode gerar pressão sob o estômago, evitar o uso de álcool e cigarro, evitar medicamentos que diminuam a pressão do esfíncter esofágico inferior, evitar roupas apertadas, e não ingerir alimentos no mínimo de três a quatro horas antes de deitar. A modificação da dieta também é importante, evitando-se alimentos como gorduras, cafeína, chocolate e cítricos, os quais geralmente desencadeiam o refluxo ${ }^{9,21}$.

O tratamento do RGE pode ser medicamentoso ${ }^{9,21}$ através de bloqueadores de hidrogênio, agentes procinéticos e inibidores de bomba de próton ${ }^{39}$. Os bloqueadores de hidrogênio atuam na diminuição do ácido gástrico. Os agentes procinéticos aceleram o esvaziamento do esôfago e estômago, produzindo maior pressão no esfíncter inferior e os inibidores de bomba de próton promovem a inibição da produção do ácido gástrico. $A$ intervenção cirúrgica também pode ser necessária em pacientes com sintomas crônicos e que não respondem satisfatoriamente ao tratamento medicamentoso $9,21,39$.

Em relação ao tratamento fonoaudiológico, este ocorrerá nos casos em que o refluxo causar alterações vocais ou até mesmo lesões laríngeas secundárias. Na presença dessas, o processo de reabilitação terá como objetivo a reabsorção, diminuição ou estabilização da lesão, a melhora da movimentação da mucosa, a melhor coaptação glótica e o uso de comportamentos vocais que não piorem as alterações já apresentadas ${ }^{9}$. 


\section{Alterações laríngeas e vocais relacionadas à regurgitação gástrica do $R L F$ e da $B N$}

O sintoma mais frequente do RLF é a sensação de globus faríngeo ${ }^{21}$, além dos relatos, na literatura, da ocorrência de disfonia, rouquidão, tosse seca, disfagia, halitose, lesões inflamatórias nas pregas vocais laringite, amigdalite, edema e eritema glótico posterior e ou de aritenóides, da região retrocricóidea e interaritenóidea, estenose subglótica, irregularidades na mucosa das pregas vocais, hipertrofia de mucosa laríngea, leucoplasia, carcinoma, laringoespasmos, granulomas, úlcera de contato, nódulos vocais, pólipos vocais, edema difuso, granuloma, fixação uni ou bilateral de aritenóides, edema de Reinke, laringomaláciaestridor, odinofagia, otalgia (explicada pela inervação sensitiva comum do esôfago com o meato acústico externo pelo nervo vago) ${ }^{8,9,21,23-25,27,28,37-41}$.

Discute-se, na literatura, se as alterações vocais provocadas pelo RLF são decorrentes de uma adaptação hiperfuncional da laringe, devido à parte distal do esôfago ser mais sensível ao ácido gástrico e, por meio do reflexo mediado pelo nervo vago, gerar tosse, pigarro, tensão, contração supraglótica e contração ânteroposterior devido à irritação $0^{8,11,36,37}$, ou se as lesões na mucosa laríngea são decorrentes do contato com o ácido gástrico devido ao comprometimento do esfíncter esofágico superior que não realiza a proteção da via aérea adequadamente ${ }^{11}$.

Os sinais e sintomas decorrentes da BN podem ser vários e graves ${ }^{7}$. Na área de cabeça $e$ pescoço, a cavidade oral pode apresentar manifestações clínicas, em decorrência dos vômitos autoinduzidos, que incluem a xerostomia, irritações da mucosa oral, sensibilidade dental à mudança de temperatura, cáries radiculares, hiperplasia das amígdalas linguais, e língua saburrosa ${ }^{38}$. As estruturas vizinhas ao esôfago também podem ser afetadas pela ação do vômito e do RLF como a via aerodigestiva superior, especificamente a laringe $\mathrm{e}^{7,10}$, a faringe, e até mesmo os pulmões, devido a aspirações, apnéia, pneumonia recorrente, asma, estenose traqueal, dentre outras complicações ${ }^{21}$.

As manifestações clínicas associadas à BN dependerão do nível, quantidade e frequência da regurgitação gástrica realizada pelo bulímico. $O$ pigarro e a tosse crônicos que surgem em reação à irritação laríngea provocada pelo RLF podem conduzir aos quadros de laringites e disfonias também chamadas de distúrbios vocais ${ }^{9}$.

A presença da disfonia do tipo orgânica em mulheres com BN tem sido relatada em estudos cujos casos clínicos apresentam queixa de rouquidão e voz grave com alterações laríngeas similares às dos portadores de $\operatorname{RLF}^{9,11}$.
Foram encontrados na literatura poucos trabaIhos relacionados à associação da BN com alterações laríngeas, sendo que os encontrados referiram apenas que a BN pode causar alterações vocais $e$ laríngeas ${ }^{11,42}$.

Um dos estudos, com oito cantoras, com tempo médio de cinco anos de BN e com idade média de 28 anos, o RLF foi encontrado em quatro delas, sendo que foi considerada uma causa adicional das alterações teciduais encontradas. Essas alterações evidenciadas pelos exames foram: edema na região pós-cricóidea (oito casos), muco espesso na laringe (oito casos), edema de pregas vocais (seis casos), hipertrofia da região glótica posterior (seis casos), obliteração do ventrículo laríngeo (seis casos), presença de vasculodisgenesia nas pregas vocais (quatro casos), e alterações polipóides nas pregas vocais (quatro casos) ${ }^{42}$.

Em outro trabalho, com 11 mulheres bulímicas que realizavam em torno de 11 episódios de regurgitação por dia, pode-se verificar as seguintes alterações: acúmulo de secreção espessa na laringe, fenda triangular médio-posterior, espessamento da mucosa na região interaritenóidea, além de pigarro (10 casos), sensação de globus faríngeo (10 casos), irritação na garganta (9 casos), quebras de sonoridade (8 casos), odinofagia (7 casos), cansaço vocal (7 casos), dentre outras ${ }^{11}$.

Dentre as possíveis alterações laríngeas decorrentes da regurgitação do ácido gástrico, podendo estar relacionadas ao RLF e à $\mathrm{BN}$, a maioria dos trabalhos evidencia: laringite posterior, úlcera de contato, granulomas, edema de Reinke, laringoespasmos, estenose subglótica, fixação uni ou bilateral de aritenóides, leucoplasia, e carcinoma ${ }^{9,28,30,38,42}$ que serão descritas a seguir.

\subsection{Laringite posterior}

A laringite é um processo inflamatório que ocorre na laringe devido a uma agressão microbiana ou irritativa ${ }^{43}$. Esta pode ser aguda proveniente de uma infecção viral ou generalizada do trato respiratório superior, caracterizada por rubor e edema de prega vocal, dor na garganta, disfonia e tem duração de alguns dias ${ }^{44}$. Na forma crônica ocorre a persistência do fator irritativo que mantém o processo inflamatório. Dentre os principais fatores etiológicos, encontram-se o tabaco, alergias, infecções rinofaríngeas e o RGE ${ }^{43}$.

A laringite mais comum causada pelo RFL é a laringite posterior que se caracteriza por eritema e espessamento da mucosa da parede posterior da laringe, conhecida por região interaritenóidea e, às vezes, por pregas vogais hipertrofiadas ${ }^{23,43,45}$. A característica da hipertrofia da mucosa interaritenóidea é a aparência reta ou abaulada em direção 
à luz laríngea (convexa), já que em situações de normalidade esta se apresenta arredondada e côncava ${ }^{45}$. Afirma-se que a falta de definição do ventrículo de Morgani devido a edema das pregas vestibulares e ao edema infraglótico são sinas frequentes de laringite devido ao RLF ${ }^{29}$.

Em um estudo com cães, nos quais foi aplicado suco gástrico na laringe, observou-se, após quatro semanas, o aparecimento de edema, eritema laríngeo, inflamação, irregularidades de mucosa e aparecimento de tecido granuloso ${ }^{9}$.

\section{2 Úlceras de contato e granulomas laríngeos}

A úlcera de contato ou úlcera da cartilagem aritenoide é uma alteração da mucosa que cobre o processo vocal das cartilagens aritenóideas, podendo ser uni ou bilateral, possuindo prevalência no sexo masculino ${ }^{46,47}$.

Dentre os principais fatores etiológicos, destacam-se a tensão muscular fonatória, ataque vocal brusco, intensidade vocal elevada e o RLF, que acaba irritando e lesando a mucosa da laringe, e causando hipersensibilidade na mucosa que, associada ao abuso vocal, pode ser considerada uma importante etiologia da úlcera de contato 47,48 .

Os principais sintomas da úlcera de contato são fadiga vocal, pigarro constante, a voz pode apresentar-se rouca ou levemente soprosa, com frequência grave, mas outros sintomas também podem estar presentes como odinofonia, desconforto na laringe, e odinofagia ${ }^{47}$.

O granuloma laríngeo é uma lesão que ocorre geralmente no processo vocal das cartilagens aritenóideas e é caracterizado pelo crescimento benigno de tecido de granulação hipertrófico ${ }^{21,45,47,49}$. Iniciase através de uma lesão na mucosa e progride para o crescimento de uma massa esbranquiçada, avermelhada ou amarelada, devido à coaptação glótica na região posterior da laringe. Pode ocorrer como progresso de uma úlcera de contato ou após uso incorreto da voz durante laringite aguda ${ }^{47}$. A etiopatogenia pode ser atribuída ao abuso vocal, RFL e intubação laríngea ${ }^{50}$.

Em relação às características vocais, a emissão é grave, tensa, com ataque vocal brusco e forte intensidade. $O$ fechamento glótico geralmente é completo, pois o granuloma localiza-se na região posterior, na porção cartilaginosa das pregas vocais, e o ataque vocal brusco acomoda os tecidos da laringe favorecendo a coaptação glótica, não ocorrendo disfonias, exceto em lesões de grande volume ${ }^{47,50}$.

Em estudo realizado com 20 homens portadores de RLF, observou-se que a alteração mais comum era a úlcera de contato e o granuloma contralateral de prega vocal ${ }^{47}$.

\subsection{Edema de Reinke}

O edema de Reinke é uma patologia laríngea caracterizada por um aumento de fluido na camada superficial da lâmina própria da mucosa das pregas vocais, logo abaixo do epitélio, também conhecida como espaço de Reinke ${ }^{47}$.

Afirma-se que a hipersensibilidade à alergia torna a mucosa mais suscetível à ação de outros fatores como abuso vocal, RLF, fumo e desordens endócrinas. A ação irritativa de algumas substâncias provoca o aumento da permeabilidade capilar dos vasos do espaço de Reinke, provocando aumento excessivo de líquidos nos tecidos e resultando em edema e hiperflacidez da prega vocal ${ }^{51}$.

As características vocais típicas são voz grave e rouca, às vezes fluida e crepitante. Em casos severos, o indivíduo pode apresentar queixa respiratória, pois o edema pode bloquear parcialmente a passagem aérea ${ }^{47,52}$.

\subsection{Laringoespasmos}

O laringoespasmo é caracterizado pela hiperadução abrupta das pregas vocais, ou seja, fechamento glótico involuntário que pode estar associado a estridor inspiratório e à obstrução do fluxo aéreo ${ }^{9}$. Em $80 \%$ dos casos adultos, o laringoespasmo deve-se ao RLF ${ }^{29}$.

Em pesquisa, utilizando cinco cachorros, observou-se que, quando aplicada solução de ácido ou ácido associado à pepsina, os cães apresentavam laringoespasmos recorrentes, indicando que o RLF é um fator causador dos laringoespasmos ${ }^{9}$.

\subsection{Estenose laríngea}

A estenose laríngea trata-se de um estreitamento cicatricial exagerado parcial ou completo da laringe ${ }^{53}$. A forma mais comum de estenose laríngea é encontrada na região subglótica da laringe e, dentre os seus fatores etiológicos, o RLF é considerado o principal causador das estenoses laríngeas nesta região e na região glótica posterior da laringe ${ }^{9,29,54}$.

\subsection{Fixação das cartilagens aritenóideas}

A fixação das cartilagens aritenóideas caracteriza-se pela redução ou impedimento completo da sua movimentação devido à corrosão da articulação cricoaritenóidea provocada pela regurgitação do ácido gástrico ${ }^{9}$.

\subsection{Leucoplasias}

São definidas como placas esbranquiçadas sobre a superfície mucosa das pregas vocais ${ }^{21,47}$, uni ou bilaterais, apresentando-se em pontos isolados ou em toda a extensão da prega vocal ${ }^{47}$. Originam-se do epitélio das pregas vocais, podendo 
penetrar na camada superficial, mas nunca no ligamento vocal enquanto benignas ${ }^{49}$. Como fatores etiológicos, destacam-se o fumo, deficiências nutricionais, RGE, e infecções nas vias aéreas superiores ${ }^{47}$.

O fechamento glótico durante os ciclos vibratórios muitas vezes é incompleto, podendo formar uma fenda irregular, os movimentos vibratórios são assimétricos, a rigidez e a massa da cobertura encontram-se aumentadas, a voz apresenta-se rouco-áspera, podendo ser bitonal e diplofônica ${ }^{47}$.

\subsection{Carcinoma de laringe}

A laringe é composta por cartilagens, tecido conjuntivo, e por um epitélio de revestimento, onde geralmente ocorre o câncer, havendo alteração do tipo celular que tem a tendência de se propagar e, caso não seja realizado o tratamento, podem ter acesso aos canais linfáticos e aos vasos sanguíneos e se distribuir por todo corpo. No caso dos carcinomas laríngeos, o sinal clínico desta propagação é o aumento dos linfonodos cervicais ${ }^{44}$.

O carcinoma epidermoide de laringe é causado principalmente pelo tabagismo e consumo excessivo de álcool concomitantemente, mas o RLF tem sido apontado como causa deste tipo de câncer ${ }^{55}$.

Ressalta-se a possibilidade de uma úlcera de contato ou uma leucoplasia progredirem para carcinoma em pacientes que apresentam DRGE em grau moderado a severo com irritação crônica da mucosa ${ }^{9}$.

Os tumores malignos de laringe representam cerca de $2 \%$ do total de câncer no Brasil, correspondendo aproximadamente a 8000 novos casos e cerca de 3000 mortes anualmente, sendo que a doença predomina em pacientes na faixa etária de 50 a 70 anos ${ }^{55}$.

\section{CONCLUSÃO}

A BN se caracteriza por um transtorno alimentar de causa principalmente psicológica em que ocorre ingestão compulsiva de grande quantidade de alimentos, seguida de episódios de vômitos autoinduzidos com a finalidade de evitar o ganho de peso. O RLF consiste em uma variação clínica da DRGE, na qual o fluxo retrógrado do alimento e do ácido gástrico entra em contato com a laringe e suas estruturas. Em comum, a BN e o RLF apresentam o mecanismo de regurgitação do ácido gástrico que pode atingir as estruturas laringofaríngeas provocando alterações vocais e laríngeas que vão das mais simples às mais significativas, podendo inclusive comprometer a qualidade de vida do indivíduo.

Dentre os sinais e sintomas laríngeos e vocais decorrentes da regurgitação do ácido gástrico sobre a laringe estão: globus faríngeo, disfonia, rouquidão, tosse seca, disfagia, halitose, lesões inflamatórias nas pregas vocais, laringite, amigdalite, edema e eritema glótico posterior e ou de aritenóides, da região retrocricóidea e interaritenóidea, estenose subglótica, irregularidades na mucosa das pregas vocais, hipertrofia de mucosa laríngea, leucoplasia, carcinoma, laringoespasmos, granulomas, úlcera de contato, nódulos vocais, pólipos vocais, edema difuso, granuloma, fixação uni ou bilateral de aritenóides, edema de Reinke, laringomalácia, estridor, e odinofagia.

Devido à escassez de literatura referente aos assuntos expostos, sugere-se que mais estudos sejam realizados, a fim de pesquisar as alterações vocais e laríngeas relacionadas diretamente à $\mathrm{BN}$, bem como o papel do próprio RLF na BN, salientando o papel do fonoaudiólogo na sua avaliação e tratamento dentro da equipe que assiste aos indivíduos com este transtorno alimentar. 


\section{ABSTRACT}

Background: characteristics of bulimia nervosa (BN) and laryngopharyngeal reflux (LPR) and laryngeal and voice disorders that can arise from exposure to gastric acid of the larynx. Purpose: to describe, through literature review, the characteristics of LPR and the BN, linking them with laryngeal and voice disorders that can stem from exposure to gastric acid of the larynx, occurring in both conditions. Conclusion: BN is characterized by an eating disorder, where there is a mainly psychological compulsive ingestion of large quantities of food, followed by episodes of self-induced vomiting in order to prevent weight gain. LPR consists of a clinical variation of gastro esophageal reflux disease (GERD), in which the retrograde flow of food and stomach acid comes into contact with the larynx and its structures. In common, BN and LPR have this mechanism of regurgitation that may reach the larynx. The signs and symptoms arising from the vocal and laryngeal regurgitation of gastric acid on the larynx are: globus pharynx, dysphonia, hoarseness, dry cough, dysphagia, halitosis, inflammatory lesions on the vocal cords, laryngitis, tonsillitis, swelling and redness and / or posterior glottis of the arytenoids, and the retrocricoid and interarytenoid region, subglottic stenosis, irregularities in the vocal folds mucosa, hypertrophy of the laryngeal mucosa, leukoplakia, carcinoma, laryngospasm, granulomas, contact ulcer, vocal nodules, vocal polyps, diffuse edema, unilateral or fixing bilateral arytenoids, edema, laryngomalacia, stridor and odynophagia

KEYWORDS: Bulimia Nervosa; Laryngeal Mucosa; Laryngopharyngeal Reflux; Eating Disorders; Voice

\section{REFERÊNCIAS}

1. Bacaltchuk J, Hay P. Tratamento da bulimia nervosa: síntese das evidências. Rev Bras Psiquiatr. 1999; 21(3):184-7.

2. Traebert J, Moreira EAM. Transtornos alimentares de ordem comportamental e seus efeitos sobre a saúde bucal na adolescência. Braz Oral Res. 2001; 15(4):359-63.

3. Claudino AM, Borges MBF. Critérios diagnósticos para os transtornos alimentares: conceitos em evolução. Rev Bras Psiquiatr. 2002; 24(3):7-12.

4. Marin VB. Trastornos de la conducta alimentaria en escolares y adolescentes. Rev Chil Nutr. 2002; 29(2):86-91.

5. Behlau M, Feijó D, Pontes P. Disfonias psiquiátricas. In: Behlau M. Voz: o livro do especialista. Rio de Janeiro: Revinter; 2005. p. 79-99.

6. Espindola CR, Blay SL. Bulimia e transtorno da compulsão alimentar periódica: revisão sistemática e metassíntese. Rev Psiquiatr RS. 2006; 28(3):265-75.

7. Balata P, Colares V, Petribu K, Leal MC. A bulimia nervosa como fator de risco para distúrbios da voz: artigo de revisão. Rev Bras Otorrinolaringol. 2008; 74(3):447-51.

8. Burati DO, Duprat AC, Eckley CA, Costa HO. Doença do refluxo gastroesofágico: análise de
157 pacientes. Rev Bras Otorrinolaringol. 2003; 69(4):458-63.

9. Behlau M, Feijó D, Pontes P. Disfonias por refluxo gastroesofágico. In: Behlau M. Voz: o livro do especialista. Rio de Janeiro: Revinter; 2005. p. 187-204.

10. Oliveira SS, Santos IS, Silva JFP, Machado EC. Prevalência e fatores associados à doença do refluxo gastroesofágico. Arq Gastroenterol. 2005; 42(2):116-21.

11. Ferreira CP, Gama ACC, Cunha CF, Santos MAR. Disfonia e bulimia: avaliação dos sintomas e sinais vocais e laríngeos. Rev Soc Bras Fonoaudiol. 2009; 14(2):177-85.

12. Vilela JEM, Lamounier MADF, Barros Neto JR, Horta GM. Transtornos alimentares em escolares. J Pediatr. 2004; 80(1):49-54.

13. Alvarenga $M$, Larino MA. Terapia nutricional na anorexia e bulimia nervosas. Rev Bras Psiquiatr. 2009; 24(3):39-43.

14. Bosi MLM, Oliveira FP. Comportamentos bulímicos em atletas adolescentes corredoras de fundo. Rev Bras Psiquiatr. 2004; 26(1):32-4.

15. Champe PC, Harve RA. Bioquímica ilustrada. Porto Alegre: Artes Médicas; 2000. p. 309-22.

16. Maso AA, Ayala MC, Rivas GZ, Mora T. Bulimia: revisión bibliográfica. Acta Odontol Venez. 2001; 39(2):70-3.

17. Gorgati SB, Holcberg AS, Oliveira MD. Abordagem psicodinâmica no tratamento dos 
transtornos alimentares. Rev Bras Psiquiatr. 2002; 24(3):44-8.

18. Yager J, Devlin MJ, Halmi KA, Herzog DB, Mitchell JE, Powers $P$, et al. Practice guideline for the treatment of patients with eating disorders. Washington, DC: American Psychiatric Publishing; 2006.

19. Castilho SM, Costa LHV. Reboxetina no tratamento da bulimia nervosa. Rev Bras Psiquiatr. 2003; 25(2):100-2.

20. Salzano FT, Cordas TA. Tratamento farmacológico de transtornos alimentares. Rev Psiquiatr Clín. 2004; 31(4):188-94.

21. Vanita A, Myron WY, Lorenz FL. Head and neck manifestations of gastroesophageal reflux disease.

Am Fam Physician. 1999; 60(3):873-86.

22. Betran O, Tagliarini VJ. Mecanismos das manifestações otorrinolaringológicas da doença do refluxo gastroesofágico. Arq Int Otorrinolaringol. 2001; 5(3):162.

23. Gomes GF, Macedo-Filho ED, Coelho JCU, Salvalaggio PRO, Sebastião APM, Gums SD. Achados histológicos na parede posterior da laringe em pacientes com refluxo gastroesofageano. Rev Bras Otorrinolaringol. 2001; 67(6):770-4.

24. Nasi A, Moraes-Filho JPP, Zilberstein B, Cecconello I, Gama-Rodrigues J. Doença do refluxo gastroesofágico: comparação entre as formas com e sem esofagite, em relação aos dados demográficos e às manifestações sintomáticas. Arq Gastroenterol. 2001; 38(2):109-15.

25. Pontes $P$, Thiago $R$, Costa $\mathrm{HO}$. Avaliação da síndrome do refluxo laringofaríngeo: estudo do limiar sensorial laríngeo. Acta Otorrinolaringol. 2007; 25(2):96-103.

26. Corsi PR, Gabliardi D, Horn M, Pochini C, Neto RMO. Presença de refluxo em pacientes com sintomas típicos de doença do refluxo gastroesofágico. Rev Assoc Med Bras. 2007; 53(2):152-7.

27. Eckley CA, Costa HO. Estudo da concentração salivar do fator de crescimento epidérmico em indivíduos com laringite crônica por refluxo laringofaríngeo. Rev Bras Otorrinolaringol. 2003; 69(5):590-7.

28. Nazar RS, Fullá JO, Varas MAP, Naser AG. Reflujofaringolaríngeo: revisión bibliográfica. Rev Otorrinolaringol Cir Cabeza Cuello. 2008; 68(2):193-8.

29. Lazarini P, Silva L. Doença do refluxo laringofaríngeo: revisão. Acta Otorrinolaringol. 2007; 25(3):190-6.

30. Soares EQW, Pinho SMR, Assencio-Ferreira VJ. Refluxo gastroesofágico: alterações laríngeas e disfonia na criança. Rev CEFAC. 2001; 3(1):45-52.
31. Montoro MA, Alcedo J. Enfermedad por reflujo gastroesofágico. Rev Esp Enferm Dig. 2004; 96(5):353.

32. Abrahao Júnior LJ, Lemme EMO, Carvalho BB, Alvariz A, Aguero GCC, Schechter RB. Relação entre o tamanho de hérnia hiatal e tempo de exposição ácida esofágica nas doenças do refluxo erosiva e não-erosiva. Arq Gastroenterol. 2006; 43(1):37-40.

33. Correa MCCSF, Lerco MM, Henry MACA. Estudo de alterações na cavidade oral em pacientes com doença do refluxo gastroesofágico. Arq Gastroenterol. 2008; 45(2):132-6.

34. Pereira GIN, Costa CDS, Geocze L, Borim AA, Ciconelli RM, Camacho-Lobato L. Tradução e validação para a língua portuguesa (Brasil) de instrumentosespecíficosparaavaliação dequalidade de vida na doença do refluxo gastroesofágico. Arq Gastroenterol. 2007; 44(2):168-77.

35. Eckley CA, Rios LS, Rizzo LV. Estudo comparativo da concentração salivar do fator de crescimento epidérmico em indivíduos com laringite crônica por doença do refluxo gatroesofágico antes e após o tratamento: resultados preliminares. Rev Bras Otorrinolaringol. 2007; 73(2):156-60.

36. Lorenzi P, Silva L. Doença do refluxo laringofaríngeo: revisão. Acta Otorrinolaringol. 2007; 25(3):190-6.

37. Capella NM, Gentil AA, Saporiti LN, Menezes AC, Nassar SM, Vicari CHF, et al. Doença do refluxo gastroesofágico: sintomas otorrinolaringológicos e achados videoendoscópicos. Arq Catarin Méd. 2002; 31(1-2):9-16.

38. Martins RHG. Manifestações otorrinolaringológicas relacionadas à doença do refluxo gastroesofágico: um tema inesgotável. Rev Bras Otorrinolaringol. 2007; 73(2):146.

39. Gavazzoni FB, Ataíde AL, Herrero Júnior F, Macedo-Filho ED. Esofagite por refluxo e laringite por refluxo: Estágios clínicos diferentes da mesma doença?. Rev Bras Otorrinolaringol. 2002; 68(1):86-90.

40. Marambaia O, Andrade NA, Varela DG, Juncal MC. Refluxo laringofaringeano: estudo prospectivo correlacionando achados laringoscópicos precoces com a phmanometria de 24 horas de 2 canais. Rev Bras Otorrinolaringol. 2002; 68(4):527-31.

41. Gurski RR, Rosa ARP, Valle E, Borba MA, Valiati AA. Manifestações extra-esofágicas da doença do refluxo gastroesofágico. J Bras Pneumol. 2006; 32(2):150-60.

42. Rothstein SG. Reflux and vocal disorders in singers with bulimia. J Voice. 1998; 12(1):89-90.

43. Huche FL, Allali A. Disfonia associada a uma laringite. In: Huche FL, Allali A. A voz: patologia 
vocal de origem orgânica. Porto Alegre: Artmed; 2005. p. 13-9.

44. Johns A. Distúrbios laríngeos em adultos. In: Fawcus M. Disfonias: diagnóstico e tratamento. 2. ed. Rio de Janeiro: Revinter; 2001. p. 120-34.

45. Cobeta I, Pérez C. Manifestações laríngeas do refluxo gastroesofágico. In: Behlau M. O melhor que vi e ouvi III: atualização em laringe e voz. Rio de Janeiro: Revinter; 2001. p. 22-30.

46. Huche FL, Allali A. Disfonias disfuncionais complicadas (com laringopatias disfuncionais). In: Huche FL, Allali A. A voz: patologia vocal de origem funcional. 2. ed. Porto Alegre: Artmed; 2005. p. 91-117.

47. Behlau M, Madazio G, Pontes P. Disfonias Organofuncionais. In: Behlau M. Voz: o livro do especialista. Rio de Janeiro: Revinter; 2001. p. 295-326.

48. Pistarino DA. Doença do refluxo gastroesofágico: conhecer para melhor tratar. [monografia]. Rio de Janeiro (RJ): CEFAC - Pós-Graduação em Saúde e Educação; 1999.
49. Mota LAA, Sefer MPC, Santos YMC. Granuloma laríngeo: tratamento fonoterápico. Odontol Clin Científ. 2003; 2(3):229-32.

50. Lemos EM, Sennes LU, Imamura R, Tsuji DH. Granuloma de processo vocal: caracterização clínica, tratamento e evolução. Rev Bras Otorrinolaringol. 2005; 71(4):494-8.

51. Abreu MHL. Edema de Reinke: aspectos gerais e tratamento. [monografia]. Rio de Janeiro (RJ): CEFAC - Pós-Graduação em Saúde e Educação; 1999.

52. Torres MLGM, Teixeira LCS. Cirurgia de edema de Reinke associado à leucoplasia. In: Behlau M. O melhor que vi e ouvi III: atualização em laringe e voz. Rio de Janeiro: Revinter; 2001. p. 297-306.

53. Ximenes-Fiho JA, Nakanishi M, Montovani JC. Estenose laríngea em crianças. Arq Int Otorrinolaringol. 2002; 6(1):181.

54. Panerari ACU, Luchi GER, Pinna B, Costa HO. Tratamento de lesões estenóticas da porção glótica anterior. Acta Otorrinolaringol. 2006; 24(3):139-45. 55 . Wunsch $\mathrm{V}$. The epidemiology of laryngeal cancer in Brazil. São Paulo Med J. 2004; 122(5):184-94.

DOI: 10.1590/S1516-18462010005000130

RECEBIDO EM: 29/11/2009

ACEITO EM: 15/06/2010

Endereço para correspondência:

Carla Aparecida Cielo

Rua Coronel Niderauer, 917/407

Santa Maria - RS

CEP: 97015-121

E-mail: fonoaud@terra.com.br 